\title{
Multiple Cavernous Hemangiomas of the Posterior Mediastinum, Lung, and Liver: A Case Report
}

\author{
Jang Hoon Lee, M.D., Young Uk Lee, M.D., Hee Joon Kang, M.D. \\ Department of Thoracic and Cardiovascular Surgery, Yeungnam University College of Medicine, Daegu, Korea
}

\section{ARTICLE INFO}

Received August 18, 2021

Revised September 13, 2021

Accepted September 28, 2021

Corresponding author

Young Uk Lee

Tel $82-53-620-3880$

Fax 82-53-626-8660

E-mail romio206@naver.com

ORCID

https://orcid.org/0000-0002-9593-4775

\begin{abstract}
A 71-year-old male patient visited Yeungnam University Hospital with abnormal chest computed tomography (CT) findings. Chest $\mathrm{CT}$ revealed multiple lung nodules and a posterior mediastinal tumor, the diagnosis of which was confirmed surgically. Magnetic resonance imaging (MRI) of the abdomen showed multiple small nodules, which were diagnosed as cavernous hemangioma in the liver based on the pathology results of the mediastinal and lung masses in combination with MRI findings. Cavernous hemangiomas are benign tumors that can occur throughout the body, mainly in the skin and subcutaneous tissue. The liver is the most common internal organ containing hemangiomas, whereas they are very rarely found in the lungs or mediastinum.
\end{abstract}

Keywords: Mediastinal neoplasms, Lung neoplasms, Hemangioma, Cavernous hemangioma, Case report

\section{Case report}

A 71-year-old male patient visited Yeungnam University Hospital with abnormal chest computed tomography (CT) findings on a routine medical examination. The patient was taking medications for hypertension, diabetes mellitus, and hypothyroidism and had a history of traumatic abdominal aortic aneurysm surgery 40 years previously. Chest CT revealed a $3.5-\mathrm{cm}$ oval mass in the posterior mediastinum at the level of the eighth to ninth thoracic vertebrae in addition to multiple pulmonary nodules (Fig. 1). Magnetic resonance imaging (MRI) of the abdomen showed multiple poorly enhanced masses in the liver. The liver masses were smaller than $2 \mathrm{~cm}$ and emitted a low T1 signal and a high T2 signal (Fig. 2A, B). We considered the possibility that the lung and liver lesions were metastatic, but blood tumor marker tests were normal. Percutaneous needle biopsy (PCNB) of the pulmonary lesion failed to yield a meaningful sample. With the possibility of neurogenic tumor or pulmonary and hepatic metastases in mind, we decided to perform surgery. We planned to resect the mediastinal tumor and biopsy the pulmonary lesion for diagnosis. We decided to determine the treatment plan for the hepatic lesions based on the surgical pathology re-
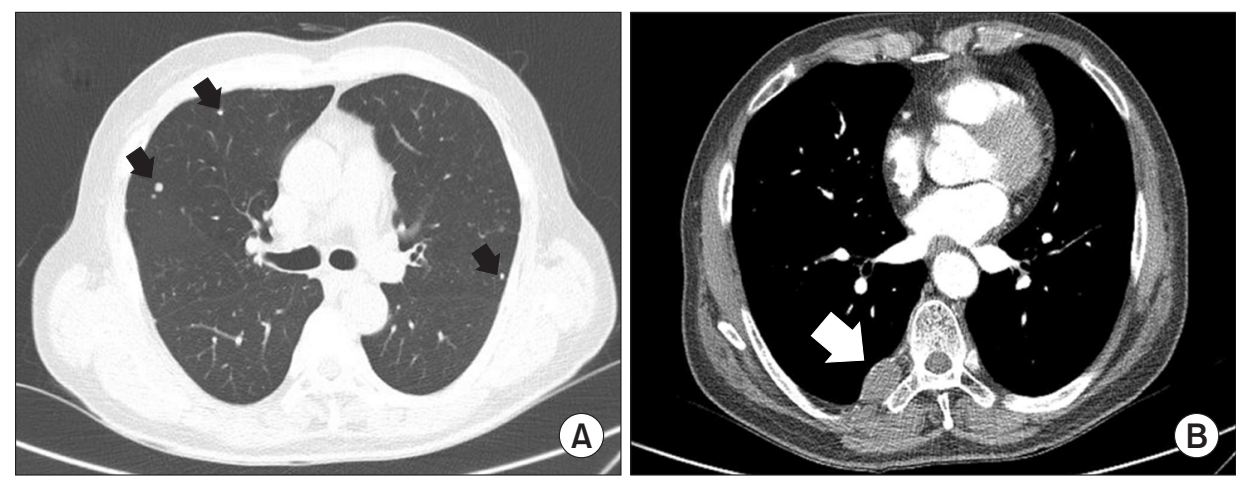

Fig. 1. Chest computed tomography (CT). (A) A preoperative chest CT reveals multiple nodules scattered in both lungs (black arrows). (B) A posterior mediastinal tumor located at the T8-9 level (white arrow).

Copyright (C) 2021, The Korean Society for Thoracic and Cardiovascular Surgery

(i) (\$) This is an Open Access article distributed under the terms of the Creative Commons Attribution Non-Commercial License (http://creativecommons.org/licenses/ by-nc/4.0) which permits unrestricted non-commercial use, distribution, and reproduction in any medium, provided the original work is properly cited. 

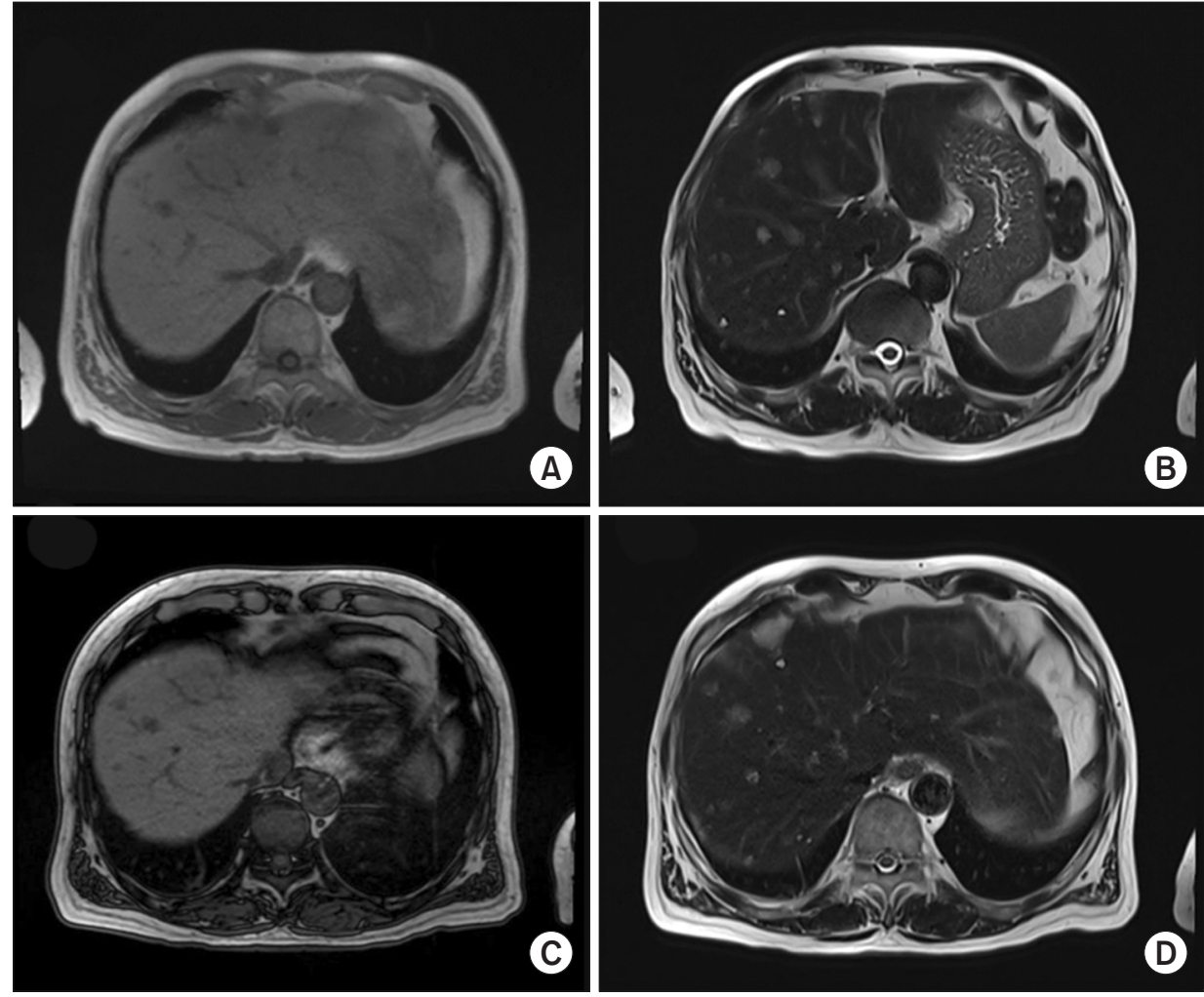

Fig. 2. Magnetic resonance imaging (MRI) of (A) the liver shows multiple small masses with a low signal on $\mathrm{T} 1$ and (B) a high signal on T2. Postoperative MRI revealed no changes in the hepatic lesions on (C) T1 or (D) T2

sults. We performed surgery with the patient in the left lateral decubitus position via video-assisted thoracoscopic surgery. We made 3 ports $(5 \mathrm{~mm}, 10 \mathrm{~mm}$, and $12 \mathrm{~mm}$ ) using a $10-\mathrm{mm}$ telescope. We identified the mediastinal tumor as observed on chest CT. The mediastinal tumor was located in the posterior mediastinum, close to the sympathetic chain and supplied by feeding vessels from the intercostal vessel. The tumor was well-demarcated without invasion into adjacent structures. After ligation and division of the feeding vessel with a hemoclip, the tumor was removed without difficulty. A pulmonary examination revealed multiple well-circumscribed nodules of different sizes, diffusely distributed on the lung surface. We performed wedge resection to obtain tissue for diagnosis. After insertion of a $28 \mathrm{~F}$ chest tube, the operation was terminated. On gross examination, the mediastinal tumor was a $3.5-\mathrm{cm}$ oval nodular mass surrounded by hardened tissue. There were 5 lung nodules in the resected lung tissue, and the cut surface showed a honeycomb-like appearance. A microscopic pathological examination revealed at both sites dilated blood vessels that varied in size and were filled with varying amounts of blood components (Fig. 3). A histopathological examination of the specimens, lung nodules, and mediastinal tumor revealed cavernous hemangioma. Based on the liver MRI and histopathology of the mediastinum and lung, we decided that the hepatic nodules were also likely cavernous hemangiomas and decided to investigate further. The patient's postoperative course was uneventful. The chest tube was removed on postoperative day 3 , and the patient was discharged on postoperative day 4. One year after surgery, chest CT and abdominal MRI revealed no changes in the pulmonary and hepatic lesions (Fig. 2C, D). The patient is doing well without any specific clinical symptoms. The patient provided written informed consent for publication of his clinical details and images.

\section{Discussion}

Cavernous hemangiomas are benign tumors that occur throughout the body, mainly in the skin and subcutaneous tissue, and also occur in the internal organs and the central nervous system [1]. The liver is the most common internal organ where hemangiomas occur, and their prevalence ranges from $0.5 \%$ to $7.0 \%$ of all cavernous hemangiomas. Cavernous hemangioma is very rarely detected in the lungs or mediastinum, as in this case [2-4]. Mediastinal cavernous hemangioma $(\mathrm{MCH})$ accounts for fewer than $0.5 \%$ of all mediastinal tumors. Nearly $70 \%$ of cases occur in the anterior mediastinum, whereas the posterior mediastinum 

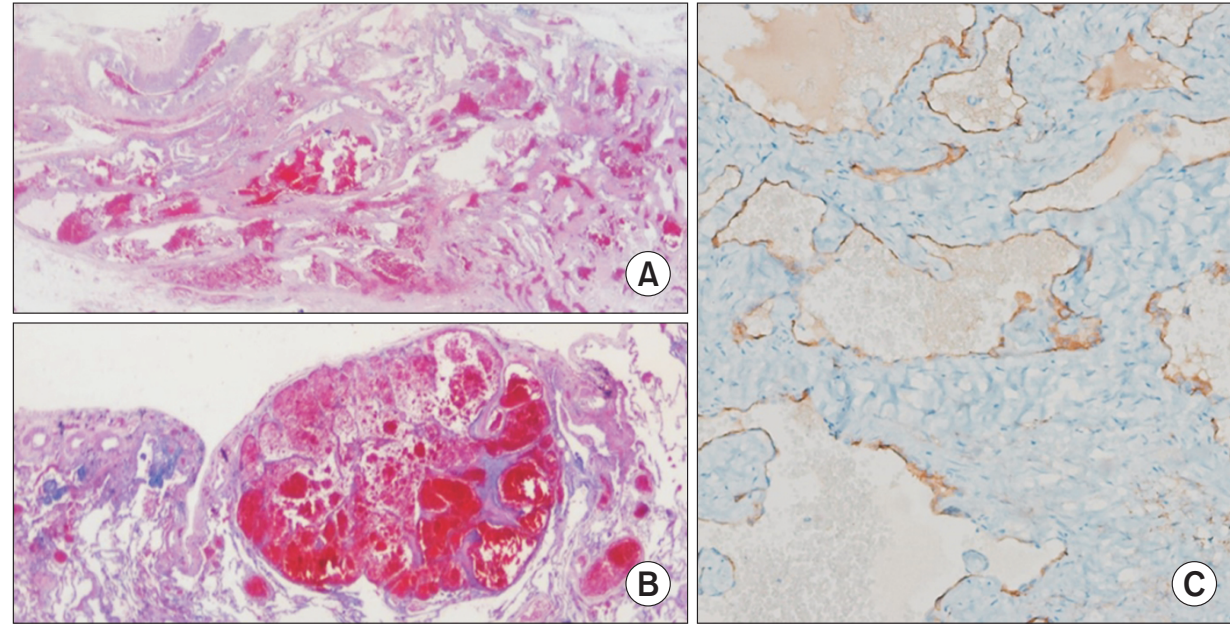

Fig. 3. Microscopic images of the mediastinal and lung cavernous hemangiomas. (A) Mediastinal hemangioma. (B) Pulmonary hemangioma. The hemangiomas consist of dilated vessels of varying size filled with blood components (H\&E, $\times 10$; scan view). (C) Immunohistochemical staining for CD34. Endothelial cells were positive for CD34 $(\times 100)$. is rarely involved [1]. A limited number of cases with mediastinal hemangioma have been reported in South Korea since the first report of MCH by Han et al. [5] in 1978, and all these cases were anterior $\mathrm{MCH}$ [6]. This case report represents the first case of posterior MCH diagnosed in South Korea. Pulmonary cavernous hemangioma $(\mathrm{PCH})$ is also rare and has been reported in only 10 to 20 cases worldwide, and only a few cases concurrently involving 3 organs have been reported worldwide [2]. The clinical symptoms of hemangiomas vary according to the organ of development, and in most cases, they are asymptomatic and detected incidentally. More than $50 \%$ of patients with mediastinal hemangioma manifest no specific clinical symptoms. Secondary symptoms depending on the site of occurrence may include cough, chest pain, dyspnea. Superior vena cava syndrome, dysphagia, and neurological symptoms are rare, but may occur [6]. Most cases of PCH are also asymptomatic. Hemoptysis is the most common and serious manifestation in symptomatic cases $[4,7]$. Depending on the site of occurrence, chest pain, shortness of breath, and pleural effusion may occur. $\mathrm{MCH}$ and $\mathrm{PCH}$ are difficult to diagnose before surgery, so most cases are diagnosed only postoperatively $[4,6]$. Although these entities are difficult to differentiate via imaging, they must be included in the differential diagnosis of mediastinal tumors that appear as clear round or oval masses. Phleboliths, blood vessel shadows, and malformed drainage veins also suggest the possibility of mediastinal hemangioma [3]. Mediastinoscopy or bronchoscopy dose not facilitate the diagnosis, and PCNB is contraindicated because of the risk of bleeding. In this case, PCNB was performed for a lung lesion but failed. This was done because the possibility of hemangioma was not taken into consideration, and closer attention to this possibility is needed in the future. The diagnosis of hepatic cavernous hemangioma $(\mathrm{HCH})$ is relatively well-known and straightforward. HCHs generally present as peripheral globular enhancements on $\mathrm{CT}$, with enhancement progressing toward the center of the nodule in the arterial and portal phases and persisting in the late phase [4]. Pathologically, $\mathrm{MCH}$ is composed of masses of varying size and dilated cavernous sinus. The inner wall is lined with vascular endothelium and the blood sinus cavity is congestive or filled with blood and occasionally phleboliths [3]. Surgical treatment is the primary treatment for cavernous hemangiomas [1-4]. Most of these masses are easily excised regardless of their location, as the borders are well differentiated from the surrounding tissues. This was also the case for our patient, as a well-defined capsulated mass with a clear border was observed in the posterior mediastinum and easily resected. If complete resection is not possible, radical resection is not recommended in the absence of recurrence or distant metastasis. Interferon- $\alpha$ is effective in treating intractable and life-threatening angiomatous disease, although it has not been used widely [4]. Studies of cavernous hemangioma involving the lung, mediastinum, and liver concurrently have yet to be published. In this case, the multiple tumors found in the liver were not histologically diagnosed, but the likelihood of $\mathrm{HCH}$ was very high considering the MRI findings and the histopathology of the lung and mediastinum. In conclusion, we operated and diagnosed clinically very rare cavernous hemangiomas that appeared simultaneously in the posterior mediastinum, lung, and livers; therefore, we report this case with a literature review. 


\section{Conflict of interest}

No potential conflict of interest relevant to this article was reported.

\section{ORCID}

Jang Hoon Lee: https://orcid.org/0000-0002-3990-888X

Young Uk Lee: https://orcid.org/0000-0002-9593-4775

Hee Joon Kang: https://orcid.org/0000-0002-4161-6616

\section{References}

1. Cohen AJ, Sbaschnig RJ, Hochholzer L, Lough FC, Albus RA. Mediastinal hemangiomas. Ann Thorac Surg 1987;43:656-9.

2. Erdogan B, Sen O, Aydin VM, Yildirim T, Bircan S, Altinors N.
Multi-organ cavernous hemangiomas: case report. Neurol Res 2003; 25:92-4.

3. Bai Y, Zhao G, Tan Y. CT and MRI manifestations of mediastinal cavernous hemangioma and a review of the literature. World J Surg Oncol 2019;17:205.

4. Zhuang BW, Li W, Chen ZF, Cao CJ, Xie XY, Xie XH. Multiple cavernous hemangiomas of the lung and liver mimicking metastasis: a case report and literature review. Medicine (Baltimore) 2018;97:e13509.

5. Hahn YS, Kim SW, Lee HK. Cavernous hemangioma of mediastinum: case report. Korean J Thorac Cardiovasc Surg 1978;11:108-11.

6. Hur J. Mediastinal cavernous hemangioma involving whole SVC: a case report. Korean J Thorac Cardiovasc Surg 2002;35:626-9.

7. Sirmali M, Demirag F, Aydin E, Karasu S, Kaya S. A pulmonary cavernous hemangioma causing massive hemoptysis. Ann Thorac Surg 2003;76:1275-6. 